\title{
Effects of silicon and germanium adsorbed on graphene
}

E. Aktürk, C. Ataca, and S. Ciraci

Citation: Appl. Phys. Lett. 96, 123112 (2010);

View online: https://doi.org/10.1063/1.3368704

View Table of Contents: http://aip.scitation.org/toc/apl/96/12

Published by the American Institute of Physics

\section{Articles you may be interested in}

High-capacity hydrogen storage by metallized graphene

Applied Physics Letters 93, 043123 (2008); 10.1063/1.2963976

Dangling-bond defects and hydrogen passivation in germanium Applied Physics Letters 91, 142101 (2007); 10.1063/1.2793184

Adsorption of carbon adatoms to graphene and its nanoribbons Journal of Applied Physics 109, 013704 (2011); 10.1063/1.3527067

Modification of electronic properties of graphene by using low-energy $\mathrm{K}^{+}$ions Applied Physics Letters 108, 181605 (2016); 10.1063/1.4948764

Graphene and related two-dimensional materials: Structure-property relationships for electronics and optoelectronics

Applied Physics Reviews 4, 021306 (2017); 10.1063/1.4983646

The role of amorphous silicon and tunneling in heterojunction with intrinsic thin layer (HIT) solar cells Journal of Applied Physics 105, 094507 (2009); 10.1063/1.3106642

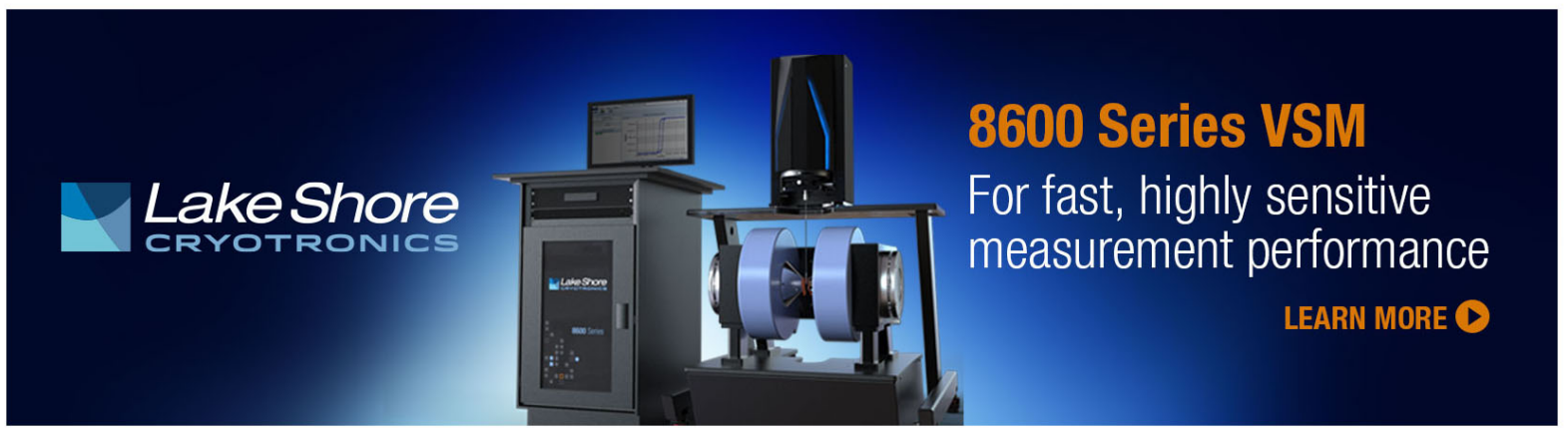




\title{
Effects of silicon and germanium adsorbed on graphene
}

\author{
E. Aktürk, ${ }^{1}$ C. Ataca, ${ }^{1,2}$ and S. Ciraci ${ }^{1,2, a)}$ \\ ${ }^{1}$ UNAM-Institute of Materials Science and Nanotechnology, Bilkent University, Ankara 06800, Turkey \\ ${ }^{2}$ Department of Physics, Bilkent University, Ankara 06800, Turkey
}

(Received 30 January 2010; accepted 2 March 2010; published online 25 March 2010)

\begin{abstract}
Based on the first-principles plane wave calculations, we studied the adsorption of Si and Ge on graphene. We found that these atoms are bound to graphene at the bridge site with a significant binding energy, while many other atoms are bound at the hollow site above the center of hexagon. It is remarkable that these adatoms may induce important changes in the electronic structure of graphene even at low coverage. Semimetallic graphene becomes metallized and attains a magnetic moment. The combination of adatom orbitals with those of $\pi$ - and $\pi^{*}$-states of bare graphene is found responsible for these effects. (C) 2010 American Institute of Physics. [doi:10.1063/1.3368704]
\end{abstract}

Two-dimensional (2D) single layer honeycomb structure of carbon, namely, graphene ${ }^{1-3}$ offers remarkable electronic and magnetic properties, which promise potential nanoscale device applications in the future. Honeycomb structure of graphene with planar $s p^{2}$-bonding and $\pi$-bonding between perpendicular $p_{z}$-orbitals underlies these unusual properties. A perfect graphene is nonmagnetic semimetal with $\pi$ - and $\pi^{*}$-bands crossing linearly at the Fermi level. Earlier studies dealing with functionalization of graphene ${ }^{4-7}$ have predicted that several atoms ( $\mathrm{Li}, \mathrm{Na}, \mathrm{K}, \mathrm{Ca}, \mathrm{Al}, \mathrm{Ga}, \mathrm{In}, \mathrm{Ti}, \mathrm{Fe}, \mathrm{Co}, \mathrm{Mn}$, etc.) are bound at the hollow site. In this work, we treated the adsorption of $\mathrm{Si}$ and $\mathrm{Ge}$, Group IV elements like $\mathrm{C}$, on graphene at different level of coverage and examined the resulting electronic and magnetic properties. We found that the unusual properties of graphene can be modified dramatically even at low $\mathrm{Si}$ and $\mathrm{Ge}$ adatom coverage. Notably, semimetallic graphene becomes metallized and attains finite spin polarization at the Fermi level.

Our predictions are obtained from the state-of-the-art spin polarized, first-principles plane wave calculations carried out within density functional theory using projector augmented-wave potentials. ${ }^{8}$ The exchange correlation functional is approximated by local density approximation. Adsorption of $\mathrm{Si}$ and $\mathrm{Ge}$ are treated in supercell geometry within periodic boundary conditions. We considered two different levels of adatom coverage $\theta$, where single $\mathrm{Si}$ or $\mathrm{Ge}$ atom is adsorbed to each $(2 \times 2)$ and $(4 \times 4)$ supercell of graphene amounting to $\theta=1 / 8$ and $\theta=1 / 32$, respectively. A plane-wave basis set with kinetic energy cutoff of $900 \mathrm{eV}$ is used. Brillouin zone (BZ) is sampled in the $\mathbf{k}$-space within Monkhorst-Pack scheme ${ }^{9}$ by $(15 \times 15 \times 1)$ and $(7 \times 7 \times 1)$ mesh points for $(2 \times 2)$ and $(4 \times 4)$ graphene cells, respectively. The convergence with respect to the number of $\mathbf{k}$-points in BZ is carefully tested. All atomic positions and lattice constants are optimized by using the conjugate gradient method, where the total energy and atomic forces are minimized. The convergence for energy is chosen as $10^{-5} \mathrm{eV}$ between two consecutive steps, the maximum Hellmann-Feynman forces acting on each atom is less than $0.04 \mathrm{eV} / \AA$ upon ionic relaxation and the pressure is kept less than $0.1 \mathrm{kBar}$. Numerical calculations are carried out by using VASP software. ${ }^{10}$

\footnotetext{
${ }^{a)}$ Electronic mail: ciraci@fen.bilkent.edu.tr.
}

Adsorption sites are determined by placing the adatom in different possible sites above graphene, such as top, T-(top of a carbon atom); bridge, B-(above a $\mathrm{C}-\mathrm{C}$ bond); hollow, $\mathrm{H}$-(above the center of hexagons) sites; and subsequently by optimizing structure to obtain minimum energy and atomic forces. The binding energies of adatoms $(\mathrm{A}=\mathrm{Si}$ or $\mathrm{Ge})$ are obtained by $E_{b}=E_{T}[$ Graphene $]+E_{T}[A]-E_{T}[$ Graphene $+A]$ in terms of the total energies of bare graphene, adatom layer after deleting graphene, and the optimized total energy of graphene $+A$ system, respectively. All energies are calculated in their magnetic state in the same supercell. The binding energy for the B site, shown in Fig. 1(a), is found to be minimum for both $\mathrm{Si}$ and $\mathrm{Ge}$. The binding energy of Si adsorbed to $(2 \times 2)$ supercell is significant and is $E_{b}$

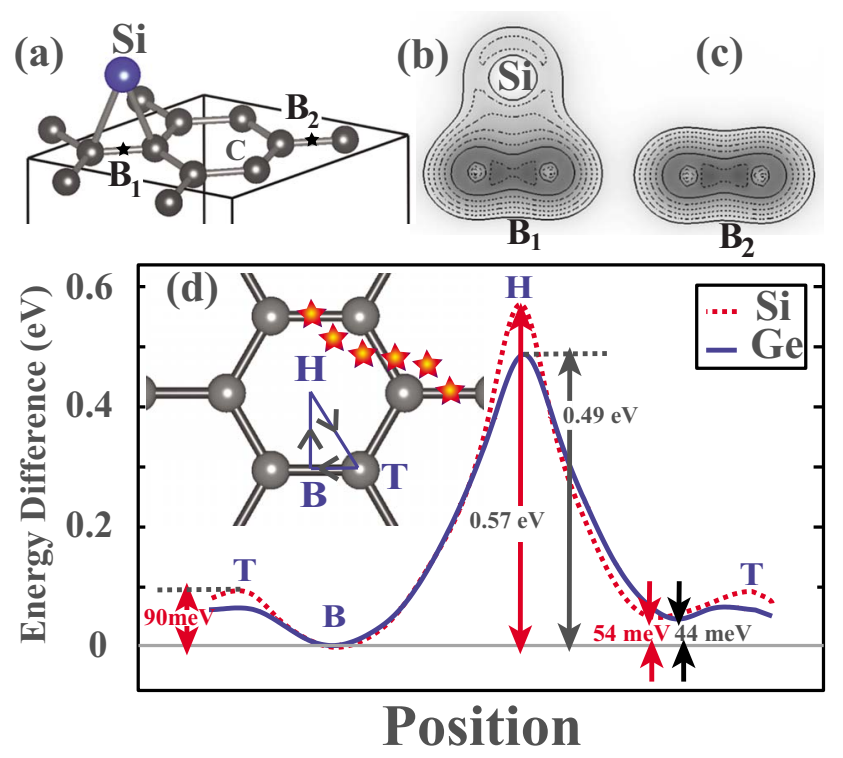

FIG. 1. (Color online) (a) Bridge site bonding geometry of Si atom adsorbed to each $(2 \times 2)$ supercell of graphene by forming bonds with the underlying $\mathrm{C}-\mathrm{C}$ bond. Large and small balls stand for Si and $\mathrm{C}$ atoms, respectively. (b) Charge density contour plots on a vertical plane passing through $\mathrm{C}-\mathrm{Si}-\mathrm{C}$, $\left(\mathrm{B}_{1}\right)$. (c) Contour plots on a different vertical plane passing through a regular $\mathrm{C}-\mathrm{C}$ bond, $\left(\mathrm{B}_{2}\right)$, without $\mathrm{Si}$ is also shown for the sake of comparison. Regular $\mathrm{C}-\mathrm{C}$ bonds have higher charge density relative to that having adsorbed $\mathrm{Si}$ above. Contour spacings are $0.02 / \AA^{3}$ electrons. (d) Variation in energy for a single $\mathrm{Si}$ and Ge adatom migrating along the path $\mathrm{T} \rightarrow \mathrm{B} \rightarrow \mathrm{H} \rightarrow \mathrm{T}$ in a $(4 \times 4)$ supercell. Zero of energy is set to the energy of the bridge site. One of the most likely path of migration is showing by stars. 
TABLE I. Calculated values for optimized structure of the single adatom $(\mathrm{A}=\mathrm{Si}$ and $\mathrm{Ge})$ adsorbed to the bridge site of each $(2 \times 2)$ and $(4 \times 4)$ supercell of graphene corresponding to the coverage $\theta=1 / 8$ and $\theta=1 / 32$, respectively. A-C distance, $d_{\mathrm{A}-\mathrm{C}}$; regular $\mathrm{C}-\mathrm{C}$ bonds in graphene, $d_{\mathrm{C}-\mathrm{C}} ; \mathrm{C}-\mathrm{C}$ distance below the adatom $A, d_{\mathrm{C}-\mathrm{C}}^{\prime}$; the lattice constant, $a$; adatom-adatom distance, $l_{\mathrm{A}-\mathrm{A}}$; binding energy, $E_{b}$ (The cohesive energy values in parenthesis are calculated relative to the free atom energies of $\mathrm{Si}$ and $\mathrm{Ge}$ in magnetic state, whereby the adatom-adatom interaction is absent); magnetic moment per supercell, $\mu$; work function, $\Phi$; dipole moment, p; charge transfer from A to graphene, $\delta q$; and spin polarization at the Fermi energy, $P\left(E_{F}\right)$. First and second rows of each adatom are for $\theta=1 / 8$ and $\theta=1 / 32$.

\begin{tabular}{lcccccccccccc}
\hline \hline & & $\begin{array}{c}d_{\mathrm{A}-\mathrm{C}} \\
\mathrm{A}\end{array}$ & $\begin{array}{c}d_{\mathrm{C}-\mathrm{C}} \\
(\AA)\end{array}$ & $\begin{array}{c}d_{\mathrm{C}-\mathrm{C}}^{\prime} \\
(\AA)\end{array}$ & $\begin{array}{c}a \\
(\AA)\end{array}$ & $\begin{array}{c}l_{\mathrm{A}-\mathrm{A}} \\
(\AA)\end{array}$ & $\begin{array}{c}E_{b} \\
(\mathrm{eV})\end{array}$ & $\begin{array}{c}\mu \\
\left(\mu_{B}\right)\end{array}$ & $\begin{array}{c}\Phi \\
(\mathrm{eV})\end{array}$ & $\begin{array}{c}\mathrm{p} \\
(\mathrm{e} \AA)\end{array}$ & $\begin{array}{c}\delta q \\
(\mathrm{e})\end{array}$ & $\begin{array}{c}P\left(E_{F}\right) \\
(\%)\end{array}$ \\
\hline \multirow{2}{*}{$\mathrm{Si}$} & $\theta=1 / 8$ & 2.04 & 1.41 & 1.45 & 4.93 & 4.93 & $1.14(1.53)$ & 0.27 & 4.06 & -0.04 & 0.32 \\
& $\theta=1 / 32$ & 2.11 & 1.41 & 1.45 & 9.80 & 9.80 & $1.17(1.17)$ & 1.02 & 4.08 & -0.03 & 0.30 & 39 \\
$\mathrm{Ge}$ & $\theta=1 / 8$ & 2.19 & 1.42 & 1.44 & 4.92 & 4.92 & $0.90(1.25)$ & 0.63 & 3.99 & -0.06 & 0.22 & -4 \\
& $\theta=1 / 32$ & 2.28 & 1.41 & 1.44 & 9.79 & 9.79 & $1.03(0.97)$ & 1.51 & 4.08 & -0.09 & 0.21 & 50 \\
\hline \hline
\end{tabular}

$=1.14 \mathrm{eV}$ with the $\mathrm{Si}-\mathrm{C}$ bond length, $d_{\mathrm{Si}-\mathrm{C}}=2.04 \AA$. Significant binding energy is achieved through the bonding combination of Si- $p_{x, y}$ and C- $p_{z}$ orbitals as well as $\mathrm{Si}-s+p_{z}$ and $\mathrm{C}-s p^{2}$ orbitals. Si atoms placed initially at hollow and top sites move to the bridge site. The adsorption of Ge having relatively larger radius is resulted in a smaller binding energy $\left(E_{b}=0.90 \mathrm{eV}\right)$ and a larger $\mathrm{Ge}-\mathrm{C}$ bond length $\left(d_{\mathrm{Ge}-\mathrm{C}}=2.19 \AA\right)$.

Despite ambiguities in defining charge transfers between atoms, we performed a Löwdin analysis ${ }^{11}$ to determine the charge transfer from $\mathrm{Si}$ or $\mathrm{Ge}$ to graphene to be $\delta q$ $\sim 0.2-0.3$ electrons. This transfer of charge attributes an ionic character to the bonding between adatom and graphene. In Figs. 1(b) and 1(c) contour plots of charge distribution of $\mathrm{C}-\mathrm{Si}-\mathrm{C}$ bridge bond and a regular $\mathrm{C}-\mathrm{C}$ bond of graphene are shown. We note that $\mathrm{C}-\mathrm{C}$ bond underlying $\mathrm{Si}$ is weakened. Structural parameters, such as the lattice constants, $\mathrm{C}-\mathrm{C}$ and $\mathrm{A}-\mathrm{C}$ bond lengths, binding energies, magnetic moments calculated for optimized $\mathrm{Si}(\mathrm{Ge})+$ graphene systems, charge transfer, work function, and electric dipole moment are given in Table I for both $\theta=1 / 8$ and $\theta=1 / 32$. The previous study investigating the interaction between $\mathrm{Si}$ and graphite surface found also that the bridge site to be one of the most energetically favorable site for single Si atom. ${ }^{12}$ The binding energy of $\mathrm{Si}$ in Table I is calculated relative to the energy of a free $\mathrm{Si}$ atom in magnetic state, since it is $\sim 0.6 \mathrm{eV}$ lower than the energy of nonmagnetic state given in Ref. 12.

In order to reveal whether the adsorbed $\mathrm{Si}$ and $\mathrm{Ge}$ can diffuse we calculate the energy barriers for the displacement of a single $\mathrm{Si}$ and $\mathrm{Ge}$ adatom in the $(4 \times 4)$ supercell along relevant paths, namely ( $\mathrm{T} \rightarrow \mathrm{B} \rightarrow \mathrm{H} \rightarrow \mathrm{T}$ ) shown in Fig. 1(d). We found that the energy barrier for the most likely path indicated in Fig. 1(d) is not high enough to prevent Si and Ge atoms from cluster formation at high temperatures.

We examined the effects of adatoms on the electronic and magnetic properties by calculating spin polarized band structure on the $(2 \times 2)$ supercell and corresponding total and projected densities of states shown in Fig. 2. For the sake of comparison we also presented the band structure of bare graphene folded to the $(2 \times 2)$ supercell. As seen in Fig. 2, bare graphene is nonmagnetic semimetal and has zero density of states at the Fermi level. The dramatic effects of adsorbed $\mathrm{Si}$ and $\mathrm{Ge}$ are clearly seen in the band structure. $\pi$ - and $\pi^{*}$-bands, which were used to cross linearly at the Fermi level, dip $\sim 1 \mathrm{eV}$ below the Fermi level upon adatom adsorption. On the other hand, the bands derived from the combination of adatom $p_{z}$ orbitals with the states of the $\pi^{*}$-conduction band (which we specify as adatom
+ graphene bands) cross the Fermi level and hence attribute a metallic character to the system. Even more remarkable is that spin-up and spin-down states of these bands split and result in a small amount of net spin in the supercell. The net
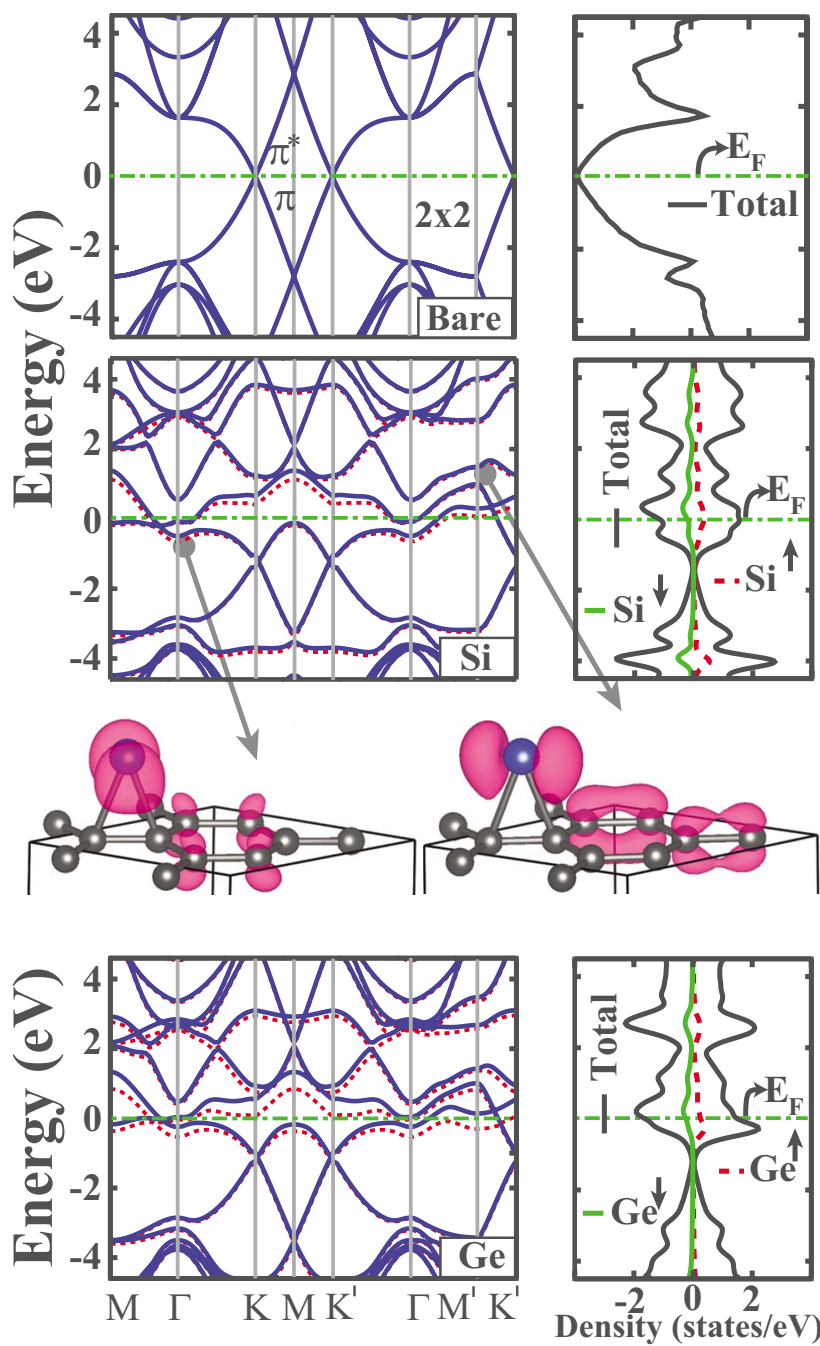

FIG. 2. (Color online) Top panels: electronic energy band structure of bare graphene folded to the $\mathrm{BZ}$ of the $(2 \times 2)$ supercell and corresponding total density of states. Zero of energy is set to the Fermi level shown by dash dotted line. Middle and bottom panels: electronic energy band structure of Si and Ge atoms periodically adsorbed to each $(2 \times 2)$ supercell of graphene at the bridge site. Spin-up and spin-down bands are indicated by dotted and solid lines, respectively. Panels on the right hand side present total (thick lines) and the adatom projected densities of states for spin-up (dashed) and spin-down (thin lines) states. Charge density isosurface of selected adatom + graphene states near the Fermi level are also shown. 


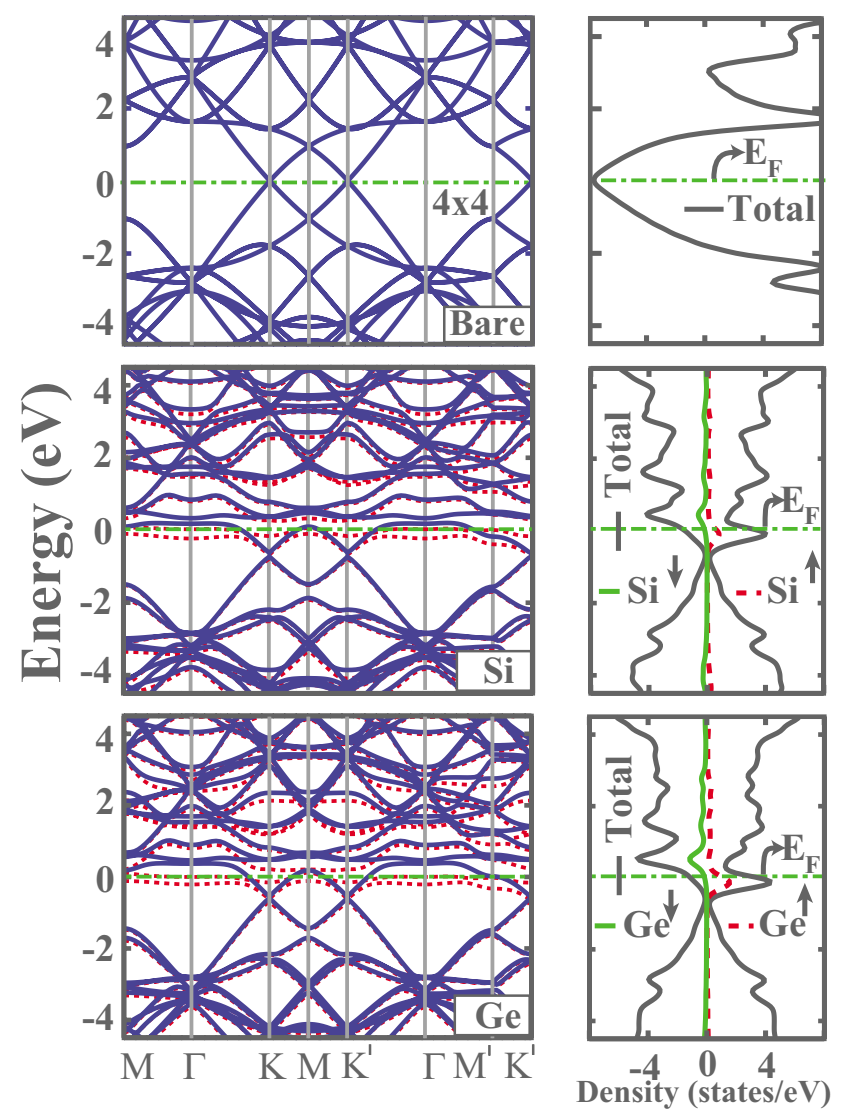

FIG. 3. (Color online) Top panels: electronic energy band structure of bare graphene folded to the $\mathrm{BZ}$ of the $(4 \times 4)$ supercell and corresponding total density of states. Middle and bottom panels: electronic energy band structure of $\mathrm{Si}$ and Ge adatoms periodically adsorbed to each $(4 \times 4)$ supercell of graphene at the bridge site. The rest of the caption is the same as Fig. 2.

magnetic moment generated for $\mathrm{Si}(\mathrm{Ge})$ adsorbed to each $(2 \times 2)$ supercell is calculated to be $0.27(0.63) \mu_{B}$ (Bohr magneton), respectively. Unbalanced spins originate from the charge transferred from adatom to graphene. The effect of adatom driven bands around the Fermi level is seen as finite density of states at the Fermi level. Note that due to breaking of spin degeneracy the density of states of different spin orientation are slightly shifted.

The adsorption of a Si or Ge atom on each $(4 \times 4)$ supercell of graphene corresponds to a coverage of $\theta=1 / 32$. In this low coverage, the adatom-adatom distance is doubled from 4.93 to $9.80 \AA$. The distance of $\sim 9.80 \AA$ is large enough to prevent adatoms from coupling. Hence, one would expect minute effects on the band structure of bare graphene; perhaps flat bands driven from the adatoms. However, the situation is rather different. The electronic energy bands and their total density of states illustrated in Fig. 3, corresponding to $\theta=1 / 32$ display similarities to those corresponding to $\theta=1 / 8$ in Fig. 2, despite the doubled adatom-adatom distance. This surprising situation occurs due to the relatively long ranged, indirect adatom-adatom coupling occurring through the combination of adatom orbitals with the $\pi$ - and $\pi^{*}$-band states of bare graphene. As a difference between $\theta=1 / 32$ and $\theta=1 / 8$, one recognizes only relatively shaper peaks in the density of states near the Fermi level due to slightly reduced dispersions of the adatom+graphene bands. This situation causes to higher spin polarization at the Fermi level, i.e., $P=\left[D\left(E_{F}, \uparrow\right)-D\left(E_{F}, \downarrow\right)\right] /\left[D\left(E_{F}, \uparrow\right)\right.$ $\left.+D\left(E_{F}, \downarrow\right)\right]$ calculated in terms of density of spin-up $(\uparrow)$ and spin-down $(\downarrow)$ states.

In summary, the adsorption of Group IV elements, $\mathrm{Si}$ and $\mathrm{Ge}$ on graphene have important and crucial consequences as follows: (i) $\mathrm{Si}$ and $\mathrm{Ge}$ are bound to the graphene at the bridge site with significant binding energies. That $\mathrm{Si}$ and Ge belong the same column in the Periodic Table, and hence they are isovalent can explain why these atoms engage in a stronger chemical interaction with graphene. In fact, C, $\mathrm{Si}$, and $\mathrm{Ge}$, all form tetrahedrally coordinated diamond crystal in three dimension. As recently predicted, not only C, but also $\mathrm{Si}$ and Ge can form stable, 2D honeycomb structure. ${ }^{13}$ (ii) Owing to relatively stronger interaction with graphene, the adsorption of $\mathrm{Si}$ and $\mathrm{Ge}$ give rise to crucial changes in electronic structure. The back donation of electrons from the adatom as well as from the underlying $\mathrm{C}-\mathrm{C}$ bond fills the adatom+graphene bands. At the end the semimetallic graphene becomes metallic with significant density of states at the Fermi level and attains a net magnetic moment. This occurs not only at high coverage but also at low coverage. Especially at $\theta=1 / 32$, the spin polarization becomes high at the Fermi level. (iii) The adatom-adatom coupling is rather long ranged. We believe that our results are relevant for various research studies on graphene.

Part of the computations have been provided by UYBHM at Istanbul Technical University through a Grant No. 2-024-2007. This work is partially supported by the project of The State Planning Organization (DPT) of Turkey and by Academy of Science of Turkey (TÜBA).

${ }^{1}$ K. S. Novoselov, A. K. Geim, S. V. Morozov, D. Jiang, Y. Zhang, S. V. Dubonos, I. V. Grigorieva, and A. A. Firsov, Science 306, 666 (2004).

${ }^{2}$ Y. Zhang, Y.-W. Tan, H. L. Stormer, and P. Kim, Nature (London) 438, 201 (2005).

${ }^{3}$ C. Berger, Z. Song, X. Li, X. Wu, N. Brown, C. Naud, D. Mayou, T. Li, J. Hass, A. N. Marchenkov, E. H. Conrad, P. N. First, and W. A. de Heer, Science 312, 1191 (2006).

${ }^{4}$ H. Sevinçli, M. Topsakal, E. Durgun, and S. Ciraci, Phys. Rev. B 77, 195434 (2008).

${ }^{5}$ K. T. Chan, J. B. Neaton, and M. L. Cohen, Phys. Rev. B 77, 235430 (2008).

${ }^{6}$ C. Ataca, E. Aktürk, S. Ciraci, and H. Ustunel, Appl. Phys. Lett. 93, 043123 (2008).

${ }^{7}$ C. Ataca, E. Aktürk, and S. Ciraci, Phys. Rev. B 79, 041406(R) (2009).

${ }^{8}$ P. E. Blöchl, Phys. Rev. B 50, 17953 (1994).

${ }^{9}$ H. J. Monkhorst and J. D. Pack, Phys. Rev. B 13, 5188 (1976).

${ }^{10}$ G. Kresse and J. Furthmuller, Phys. Rev. B 54, 11169 (1996).

${ }^{11}$ P.-O. Löwdin, J. Chem. Phys. 18, 365 (1950).

${ }^{12}$ J. H. Wu and F. Hagelberg, Phys. Rev. B 76, 155409 (2007).

${ }^{13}$ S. Cahangirov, M. Topsakal, E. Aktürk, H. Şahin, and S. Ciraci, Phys. Rev. Lett. 102, 236804 (2009). 